\title{
UNE MÉTHODE QUASI-NEWTON POUR LE CALCUL D'ÉCOULEMENTS VISCOÉLASTIQUES.
}

\author{
THIERRY DUMONT.
}

\begin{abstract}
On présente ici une méthode pour le calcul numérique d'écoulements de fluides viscoélastiques de type différentiel. Le système non linéaire obtenu après discrétisation est résolu à l'aide de la méthode GMRES. Il en résulte une méthode économique. Des résultats numériques sont présentés, où l'on met particulièrement l'accent sur l'étude des tourbillons.
\end{abstract}

Mots-clés : Viscoélasticité, FENE-P, Oldroyd-B, PTT, GMRES. Classification mathématique: 65N30, 76D99, 76M10.

\section{INTRODUCTION}

Le calcul numérique d'écoulements de fluides viscoélastiques pose plusieurs problèmes difficiles. Si les premiers obstacles apparus - problèmes de compatibilité entre les discrétisations des différents champs, caractère convectif des équations de comportement - sont maintenant bien compris, ces calculs restent extrêmement coûteux en temps machine et en espace mémoire, de part la forte non-linéarité des problèmes et parce qu'il faut approcher 3 champs dont l'un — celui des contraintes - est forcément particulièrement demandeur de place mémoire. Ici, on a cherché systématiquement des méthodes de discrétisation peu encombrantes mais précises, et des algorithmes performants mais simples à implanter. Il en résulte un code de calcul assez rapide qui devrait sans trop de problèmes et sans surcoût excessif s'adapter à des modèles assez réalistes (comme les modèles à plusieurs temps de relaxation par exemple).

L'étude des recirculations dans des domaines simples est, avec la comparaison entre les contraintes calculées et celles mesurées un moyen important de valider un modèle face à la réalité expérimentale : ce sont les principaux résultats numériques présentés ici.

\section{Modèles Mathématiques}

Les inconnues sont $u$ le champ des vitesses, $p$ celui des pressions et $T$ celui des extra-contraintes.

On considère tout d'abord les modèles classiques d'Oldroyd et de PhanThien et Tanner (P.T.T.) .

On note $D u=\frac{1}{2}\left(\nabla u+\nabla u^{t}\right)$ le tenseur des vitesses de déformation, $B(\tau, u)=\tau \nabla u+\nabla u^{t} \tau$.

Les deux modèles sont décrits par les équations (1) à (4) :

$$
\begin{gathered}
\operatorname{Re}(u \cdot \nabla) u-\operatorname{div} T+\operatorname{grad} p=0, \\
\nabla \cdot u=0,
\end{gathered}
$$




$$
T=\tau+2(1-\eta) D u
$$

La composante viscoélastique $\tau$ des extracontraintes est solution de l'équation de comportement :

$$
Y(\varepsilon, \tau) \tau+\lambda\left(\frac{\partial \tau}{\partial t}+(u . \nabla) \tau-B(\tau, u)\right)-2 \eta D u=0
$$

avec :

$Y(\varepsilon, \tau)=1$ (pour le modèle d'Oldroyd).

et

$Y(\varepsilon, \tau)=\exp \left(\frac{\varepsilon \lambda}{\eta} \operatorname{trace} \tau\right)$ pour le modèle P.T.T. $(\varepsilon \geq 0)$

Re $\geq 0$ est le nombre de Reynolds, en général voisin de $0, \lambda \geq 0$ un temps de relaxation. $\eta$ représente la proportion de matériau viscoélastique dans le fluide $(0 \leq \eta \leq 1)$.

Pour les conditions aux limites, on impose $u$ sur la frontière $\Gamma$ du domaine $\Omega$ de l'écoulement ${ }^{1}$, et on impose $\tau$ sur $\Gamma_{-}$, partie de $\Gamma$ où le champ des vitesses est rentrant dans $\Omega$.

On considérera aussi le modèle FENE-P [1] : l'équation (4) est remplacée par :

$$
\tau=\frac{\eta f(\operatorname{trace} A)}{\lambda} A
$$

où $A$ est donné par :

$$
\left.\frac{\partial A}{\partial t}+(u . \nabla) A-B(A, u)\right)-f(\operatorname{trace} A) \frac{A-I}{\lambda}=0,
$$

avec :

$$
f(x)=\frac{x}{1-\varepsilon x} \varepsilon \geq 0 .
$$

Remarque 1. A l'aide de l'équation de comportement (4)(ou (5),(6) et (7)) on peut considérer $\tau$ comme une fonction de $u: \tau=\tau(u)$.

Alors (1) (2) (3) donnent :

$$
\begin{aligned}
\operatorname{Re}(u . \nabla) u-2(1-\eta) \nabla \cdot D u+\nabla p-\nabla \cdot \tau(u) & =0, \\
\nabla . u & =0 .
\end{aligned}
$$

C'est ce système non-linéaire qu'on va résoudre après discrétisation.

\footnotetext{
${ }^{1}$ éventuellement des conditions d'écoulement établi en sortie (voir page 177).
} 


\section{DiscrÉtisation PAR ÉLÉMENTS FINIS}

Soient $U_{h}, P_{h}, \mathrm{~T}_{h}$ les espaces d'approximation pour $u, p, \tau$. La discrétisation pose 2 types de problèmes:

1. la compatibilité des champs: les approximations de $u$ et de $p$ doivent vérifier la classique condition Inf-Sup du problème de Stokes [2] ; d'autre part $\tau$ et $u$ doivent aussi vérifier une condition Inf-Sup [4]. On peut donc pour prendre pour $u$ et $p$ n'importe quelle approximation admissible du problème de Stokes. Pour $\tau$, il faut soit utiliser des approximations continues à grand nombre de degrés de liberté (ce qui est forcément très coûteux) soit choisir des espaces $U_{h}$ et $\mathrm{T}_{h}$ tels que $\mathrm{T}_{h} \subset D U_{h}$ (ce qui implique pratiquement une approximation discontinue pour $\tau)[4]$.

2. le caractère convectif de l'équation de comportement impose l'utilisation d'une forme de décentrement pour assurer la stabilité des solutions.

Ici on a choisit une discrétisation en éléments finis triangulaires, avec pour $U_{h}$ des éléments finis $P_{2}^{+}$continus (c'est l'élément $P_{2}$ auquel on a ajouté dans chaque triangle la bulle $\left.=27 \lambda_{1} \lambda_{2} \lambda_{3}\right)$ et pour $P_{h}$ des élements finis P1 discontinus [2]. Pour $\mathrm{T}_{h}$ nous avons utilisé des éléments finis P2 discontinus pour chaque composante du tenseur avec, pour l'équation de comportement, la formulation de Lesaint-Raviart [3] :

Trouver $\tau \in \mathrm{T}_{h}$ tel que, pour tout élément $E \mathrm{du}$ maillage :

$$
\begin{aligned}
\int_{E}(Y(\varepsilon, \tau) \tau & +\lambda((u . \nabla) \tau-B(\tau, u))-2 \eta D u): S+ \\
& +\int_{\partial E_{-}}[\tau] u . n_{E} . S=0 \quad \forall S \in T_{h},
\end{aligned}
$$

où $[\tau]$ est le saut de $\tau$ à travers la frontière $\partial E$ de l'élement, $n_{E}$ est la normale unité sortante à $\partial E$ et $\partial E_{-}$est la partie de $\partial E$ où $u . n_{E}<0$. Au bord $\Gamma_{-}$, les conditions aux limites sont introduites à l'aide du saut $[\tau]$. (9) s'adapte immédiatement au modèle FENE-P.

La formulation variationnelle discrète des équations (1),(2) et (3) est classique :

Trouver $u \in U_{h}, p \in P_{h}, u$ verifiant les conditions aux limites tels que :

$$
\begin{gathered}
2(1-\eta) \int_{\Omega}\{D u: D v-p \nabla \cdot v\}+\int_{\Omega} \tau \nabla v=0 \quad \forall v \in\left\{U_{h} ;\left.v\right|_{\Gamma}=0\right\} \\
\int_{\Omega} q \nabla \cdot u=0 \quad \forall q \in P_{h}
\end{gathered}
$$

(9),(10) et (11) définissent le problème discrétisé.

En fonction de la taille $h$ des éléments, l'erreur entre la solution calculée et la solution exacte vérifie (c.f. [10]) :

$$
\| u-\left.u_{\text {exact }}\right|_{H^{1}(\Omega)^{2}}+\left|p-p_{\text {exact }}\right|_{L^{2}(\Omega)}+\left|\tau-\tau_{\text {exact }}\right|_{L^{2}(\Omega)^{4}}=0\left(h^{2}\right)
$$




\section{RÉSOlution DES PROBlÈmes DiscrétisÉS}

D’après la REMARQue 1 le problème discret s'écrit comme un problème non-linéaire en $u$ et $p$. Les différentes méthodes de point fixe envisageables ne convergent que pour des fluides très peu élastiques; la méthode de Newton est extrêmement coûteuse et difficile à implanter. Il semble plus intéressant d'utiliser une méthode Quasi-Newton comme la méthode GMREs [5].

\subsection{GMRES EN QUELQUES MOTS}

Soit $F(X)=0$ un système non linéaire. La méthode de Newton consiste à construire une suite $X_{0} \ldots X_{n}, X_{n+1}$ en résolvant à chaque pas le système $D F\left(X_{n}\right)\left(X_{n}-X_{n+1}\right)=F\left(X_{n}\right)$.

Deux idées permettent de construire une méthode Quasi-Newton [5] :

1. résoudre approximativement le système précédent en effectuant $q$ itérations d'une méthode itérative de type Krylov qui n'utilise que des produits matrice-vecteur (la méthode du Résidu Minimal Généralisé, GMRES) ;

2. puisque seuls des produits matrice-vecteur sont nécéssaires, (i.e. les produits de la forme $\left.D F\left(X_{n}\right) . W\right)$, on les approche par des différences finies : $D F\left(X_{n}\right) . W \simeq \frac{1}{h}\left(F\left(X_{n}+h . W\right)-F\left(X_{n}\right)\right)=D_{h} F\left(X_{n}\right) . W$.

Il n'y a donc pas besoin de construire la matrice jacobienne et il suffit de savoir évaluer $F$.

Il faut stocker $q+1$ vecteurs : $\left\{W, D F_{h}\left(X_{n}\right) \cdot W, \ldots, D F_{h}\left(X_{n}\right)^{q} . W\right\}$. Les seules difficultés pratiques sont le choix de $h$ et celui de $q$ (qu'on espère évidemment pouvoir prendre petit...). La méthode peut être améliorée pour en faire une méthode de descente pour $\|F(X)\|^{2}$ (c'est la méthode utilisée ici (c.f. [5])).

\subsection{L'Algorithme}

Définissons $F$ et $X$ pour notre problème ; suivant la Remarque 1, on choisit $X=(u, p)$.

L’idée consiste à préconditionner (8) par un problème de Stokes:

Soit $(W, \Pi) \in U_{h} \times P_{h},\left.W\right|_{\Gamma}=0$ solution de :

$$
\begin{gathered}
2 \int_{\Omega}\{D W: D v-\Pi \operatorname{grad} . v\}= \\
2(1-\eta) \int_{\Omega}\{D u: D v-p \operatorname{grad} . v\}+\int_{\Omega} \tau(u) \operatorname{grad} v \\
\forall v \in\left\{U_{h} ;\left.v\right|_{\Gamma}=0\right\} \\
\int_{\Omega} q \operatorname{grad} . W=0 \quad \forall q \in P_{h}
\end{gathered}
$$

Il est immédiat que si $W$ et $\Pi$ sont tous deux nuls, alors $(u, p, \tau(u))$ sont solutions du problème discret. On prend donc $F:(u, p) \mapsto(W, \Pi)$.

En pratique, il suffit donc de savoir calculer $F(u, p)$. Pour cela, il faut :

1. Calculer $\tau$ à l'aide de (4) (ou (5),(6) et (7)) ;

2. Calculer le second membre de (12); 


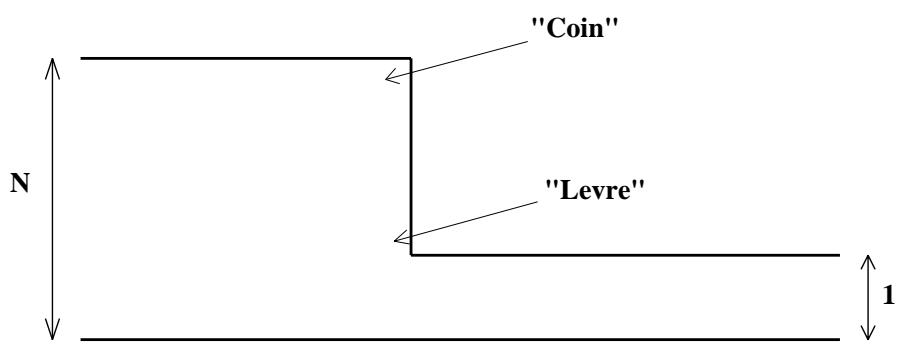

Figure 1. Contraction N:1 (domaine de calcul (longueur totale $=50)$; le domaine réel est symétrique autour de l'axe horizontal).

3. Résoudre le problème de Stokes (12),(13).

4.2.1. Calcul de $\tau$. Dans le cas du fluide d'Oldroyd-B, l'équation de comportement (9) est linéaire et on obtient un système linéaire de la forme $\left(A_{s}+A_{b}\right) \tau=f ; A_{s}$ (formée de blocs diagonaux d'ordre 18) provient des intégrales de surface sur chaque élément et $A_{b}$ des intégrales sur les frontières des éléments. Pour les autres modèles, $A_{s}=A_{s}(\tau)$ est non linéaire. On utilise une relaxation par blocs (linéaire ou non linéaire selon le modèle) à l'aide des blocs de $A_{s}$ en balayant les éléments. Pour les modèles P.T.T ou FENE-P, il faut résoudre les systèmes par la méthode de Newton sur chaque élément. Une renumérotation des éléments permet d'obtenir la convergence en un très petit nombre d'itérations [6].

Remarque 2. La méthode GMRES a été utilisée par A. Fortin et ses collaborateurs [8] [9], tout d'abord en l'appliquant au système complet d'équations, puis en éliminant $u$ et $p$; l'élimination de $\tau$ proposée ici devrait permettre de résoudre des problèmes à plusieurs temps de relaxation sans augmentation de la taille mémoire nécéssaire.

\section{Simulations numÉriques}

Sauf mention contraire, les simulations ont été réalisées avec $\operatorname{Re}=0$ et $\eta=8 / 9$.

On a considéré des écoulements dans des contractions N:1 (voir figure 1).

Le nombre de Weisenberg $W e$ est définit comme $W e=\lambda \dot{\gamma}$ où $\dot{\gamma}$ est le taux de cisaillement mesuré à la frontière de la partie aval, dans l'écoulement établi. La composante verticale de $u$ est fixée à 0 sur l'axe de symétrie horizontal et à la sortie. En entrée, les vitesses et les contraintes sont celle d'un écoulement de Poiseuille. En sortie, le tenseur de Cauchy $\sigma=-p I d+T$ vérifie $(\sigma . n) . n=0$ (conditions d'écoulement établi).

\subsection{Nombre de Weissenberg maximum}

Pour le modèle d'Oldroyd (figure 2), il se situe autour de $W e=10$ dans les contractions $4: 1,8: 1$ et $16: 1$. Il s'avère impossible d'améliorer cette limite en raffinant le maillage et, en fait, la limite diminue quand la taille des éléments décroit. La perte de convergence se manifeste par l'apparition de fortes oscillations en $\tau$ au voisinage du coin réentrant. Avec les modèle P.T.T. et FENE-P $(\varepsilon=0.02)$, on ne rencontre pas de telles limitations et 
on obtient des résultats stables jusqu'à $W e=20$. Il semble qu'on peut améliorer ce résultat en allongeant le tube sortant et le nombre d'éléments.

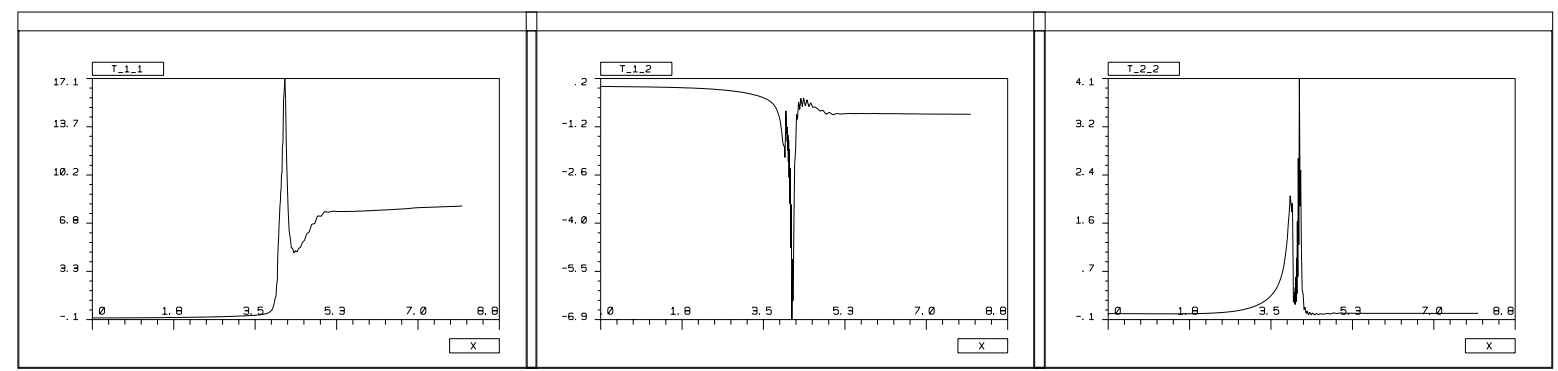

Figure 2. Modèle d'Oldroyd, contraction 4:1, We=5. Coupes horizontales de $\tau_{11}, \tau_{12}$ et $\tau_{13}$ vers le coin réentrant $(\mathrm{de}(\mathrm{x}=-4, \mathrm{y}=1)$ à $(\mathrm{x}=4, \mathrm{y}=1)$, le coin réentrant étant en $(1,1))$

\subsection{Tourbillons}

5.2.1. Modèle D'Oldroyd. Le tourbillon de coin est peu modifié par rapport au fluide Newtonien; un tourbillon de lèvre apparait dans la contraction 4:1 quand $W e \geq 4$. Ce tourbillon décroit dans les contractions 8:1 et 16:1. Ceci conforte les arguments de Rallison et Hynch [7], ainsi que les expériences numériques de Keiller [1] qui montrent qu’il ne doit pas y avoir de tourbillon dans le cas d'un fluide s'écoulant dans un puits.

5.2.2. Modèle P.T.T.. Avec $\varepsilon=0.02$ un tourbillon de lèvre apparait dès que $W e \geq 4$. Les 2 tourbillons croissent avec $W e$ et, à partir de $W e=12$, la fonction courant ne possède plus qu'un maximum et le mélange des 2 tourbillons est complet (figure 3).

L'intensité des tourbillons (maximum de $|\psi|, \psi$ est la fonction courant) en fonction de $W e$ est résumée dans le tableau suivant :

\begin{tabular}{|c|c|c|}
\hline$W e$ & Lèvre & Coin \\
\hline 5 & $8.810^{-5}$ & $2.810^{-4}$ \\
\hline 6 & $2.610^{-4}$ & $2.8610^{-4}$ \\
\hline 8 & $3.710^{-4}$ & $3.110^{-4}$ \\
\hline 12 & $1.010^{-3}$ & $710^{-4}$ \\
\hline
\end{tabular}

\begin{tabular}{|c|c|}
\hline$W e$ & intensité \\
\hline 13 & $1.310^{-3}$ \\
\hline 14 & $1.610^{-3}$ \\
\hline 17 & $2.910^{-3}$ \\
\hline
\end{tabular}

Dans la contraction $16: 1$, les 2 tourbillons ne se mélangent évidemment pas. Le tourbillon de lèvre croit avec $W e$ : 


\begin{tabular}{|c|c|}
\hline$W e$ & Intensité du tourbillon de lèvre \\
\hline 5 & $8.010^{-5}$ \\
\hline 6 & $1.010^{-4}$ \\
\hline 8 & $1.610^{-4}$ \\
\hline 10 & $3.410^{-4}$ \\
\hline 12 & $4.410^{-4}$ \\
\hline
\end{tabular}
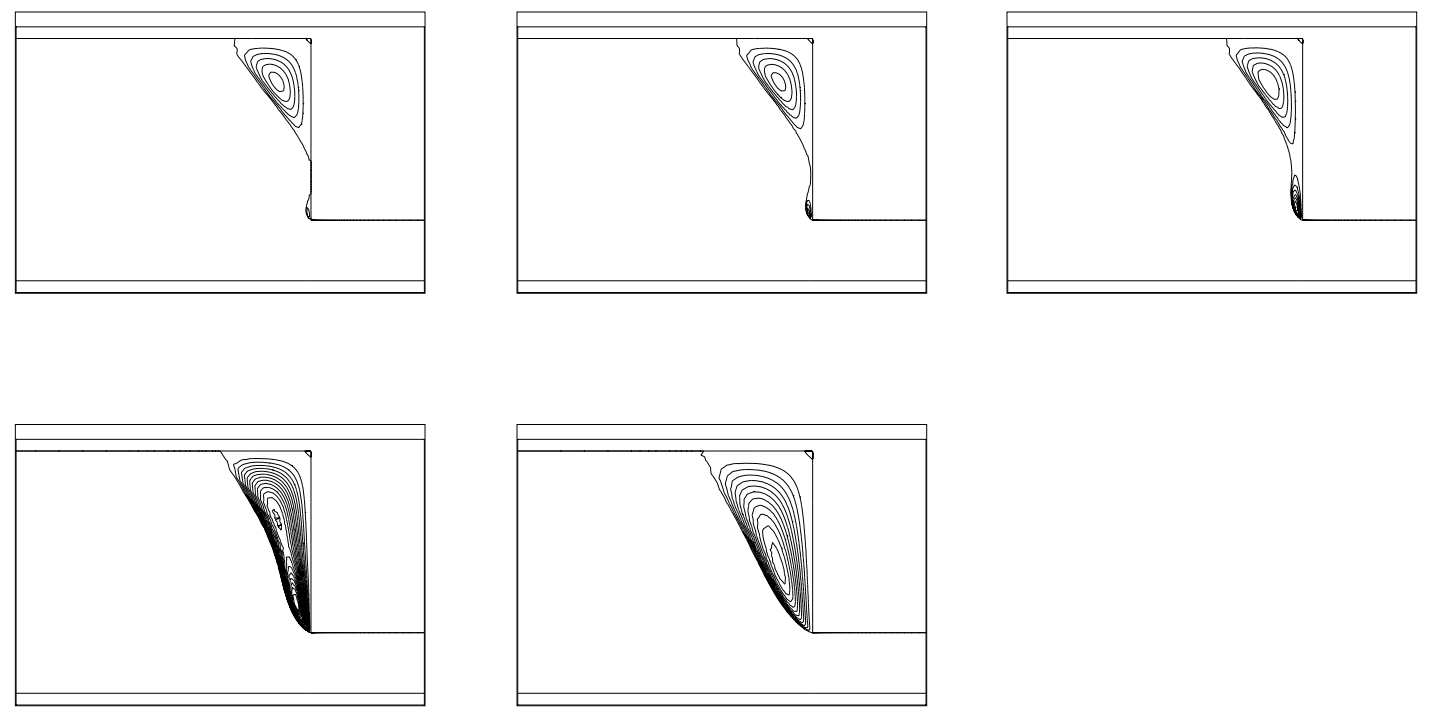

Figure 3. Modèle P.T.T., contraction 4:1, $W e=5,6,8,12$ et 17

5.2.3. ModèLE FENE-P. Les calculs dans une contraction 16:1 montrent des recirculations très voisines de celles qu'a obtenu Keiller [1] dans un domaine non borné de frontière régulière, avec apparition d'un tourbillon de lèvre qui croit avec We (figure 4) :

\begin{tabular}{|c|c|}
\hline$W e$ & Intensité du tourbillon de lèvre \\
\hline 4 & $2.310^{-5}$ \\
\hline 8 & $1.210^{-4}$ \\
\hline 10 & $2.310^{-4}$ \\
\hline
\end{tabular}
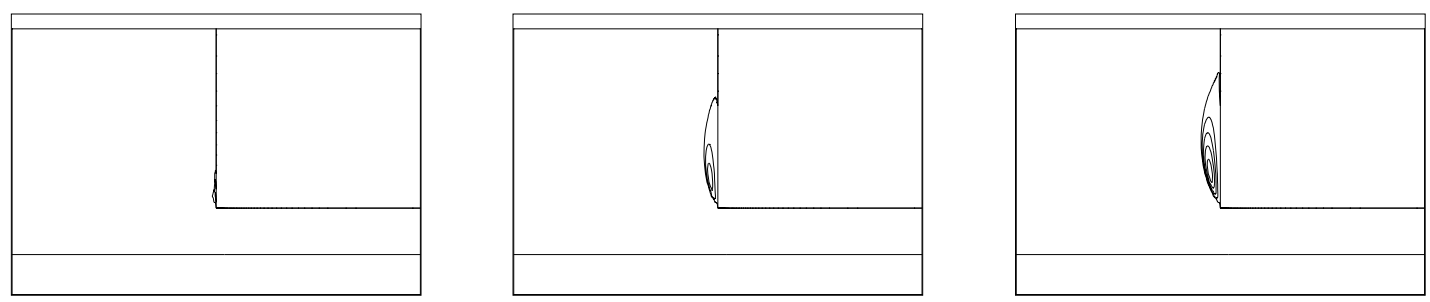

Figure 4. Modèle FENE-P, contraction $4: 1, W e=4,8$ et 10 
5.2.4. Prise en compte de L'inertie. Si $R e>0$ les effets les plus visibles concernent l'intensité des tourbillons et la chute de pression. Les résultats cidessous concernent le fluide de type P.T.T. dans la contraction $4: 1(\varepsilon=0.02$, $\eta=8 / 9)$.

LES TOURBILLONS : on observe une réduction de la taille et de l'intensité des tourbillons de coin et de lèvre quand $R e$ croit (voir également la figure 6) :

\begin{tabular}{|l|c|c|c|c|}
\hline & \multicolumn{2}{|c|}{$W e=5$} & \multicolumn{2}{c|}{$W e=12$} \\
\hline & Coin & Lèvre & Coin & Lèvre \\
\hline $\operatorname{Re}=0$ & $0.2810^{-3}$ & $0.9210-4$ & $0.710^{-3}$ & $0.1010^{-2}$ \\
\hline $\operatorname{Re}=1.25$ & $0.1810^{-3}$ & $0.6810^{-4}$ & $0.3310^{-3}$ & $0.810^{-3}$ \\
\hline $\operatorname{Re}=2.5$ & $0.1210^{-3}$ & $0.4910^{-4}$ & $0.1510^{-3}$ & $0.6410^{-3}$ \\
\hline $\operatorname{Re}=5$ & $0.6010^{-5}$ & $0.4510^{-4}$ & $0.5910^{-4}$ & $0.4210^{-4}$ \\
\hline
\end{tabular}

Figure 5. Intensité des tourbillons modèle PTT; 4:1, (max. $|\psi|)$
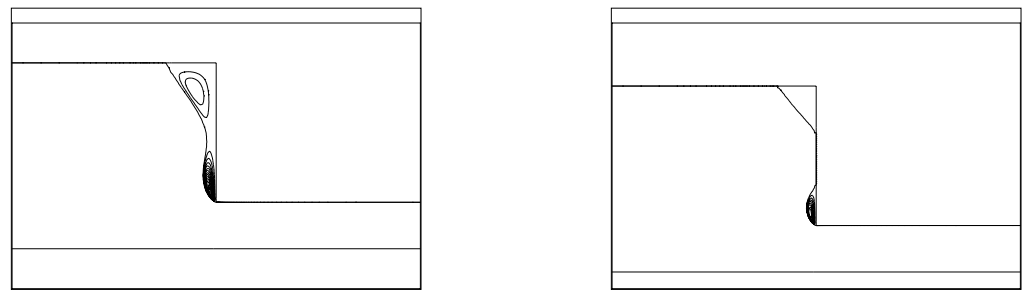

Figure 6. Modèle P.T.T. ; influence de l’inertie ( $R e=2.5$ et 5) 
LA CHUTE DE PRESSION :

Suivant Keiller [1], on définit une chute de pression normalisée comme la différence entre la chute de pression mesurée dans le domaine et celle obtenue pour un fluide Newtonien, divisée par la chute de pression par unité de longueur dans le canal sortant pour un fluide Newtonien.

On observe une diminution de cette quantité quand Re augmente, mais c'est un phénomêne bien moins important que la chute due à la viscoélasticité :

\begin{tabular}{|c|c|c|}
\hline & \multicolumn{2}{|c|}{ Chute de pression normalisée } \\
\hline $\operatorname{Re}$ & $W e=5$ & $W e=12$ \\
\hline 0 & 7.69 & 13.47 \\
\hline 1.25 & 7.59 & 13.44 \\
\hline 2.5 & 7.52 & 13.41 \\
\hline 5 & 7.45 & 13.3 \\
\hline
\end{tabular}

5.2.5. QUELQUES DONNÉES CONCERNANT LES EXPÉRIMENTATIONS NUMÉRIQUES.

- Tous les calculs ont été effectués avec un maillage d'environ 2000 éléments pour un total de 4000 nœuds, ce qui donne 36000 inconnues pour les contraintes, mais seulement 14000 pour chaque vecteur de GMRES : l'élimination de $\tau$ est donc très économique du point de vue mémoire.

- La taille $q$ de l'espace de Krylov a été choisie entre 10 et 20 avec une valeur d'autant plus grande que We est grand.

- Le choix du paramètre $h$ pour le calcul de dérivées approchées n'est pas très critique : on a pris $h=C\|X\|$ (norme dans $\mathbb{R}^{n}$ ) avec $C$ entre $10^{-6}$ et $10^{-8}$ sans modification sensible des performances de l'algorithme.

- A chaque pas, le plus cô̂teux en temps cPu est le calcul de $\tau$. En comparaison, la résolution des problèmes de Stokes - qui ont des matrices constantes- est négligeable.

- Tous les calculs ont été effectués sur un calculateur HP730 avec 32 Mo de mémoire. Le temps CPU nécéssaire pour ateindre $W e=17$ est le l'ordre de 4 heures avec le modèle P.T.T..

\section{Conclusion}

L'apport principal de cette méthode semble bien être l'économie de moyens informatiques qu'elle permet.

En ce qui concerne le modèle d'Oldroyd, les limites en nombre de Weissenberg n'ont pas pu être repoussées et la décroissance du nombre de Weissenberg limite avec la taille des éléments suggère que la solution — si elle existe dans la contraction 4:1- n'est pas suffisamment régulière pour être approchée par des éléments finis.

La généralisation à d'autres lois différentielles ne poserait pas de problème et la méthode serait sans doute intéressante pour des problèmes à plusieurs temps de relaxation.

En dimension 3, la méthode Quasi-Newton serait surement avantageuse, à condition bien sur de pouvoir utiliser des discrétisations encore beaucoup moins coûteuses. 


\section{REFERENCES}

[1] KEILLER R.A. Entry-flow calculations for the Oldroyd-B and FENE equations J.N.N.F.M. 46, PP 143-178 (1993)

[2] Girault V.,Raviart P.A Finite Element Method for Navier-Stokes equations. Theory and Algorithms SPRINGER (1986)

[3] Lesaint P., RAViaRT P.A. On a finite element method for solving the neutron transport equation Mathematical Aspects of Finite Elements in Partial Differential Equations, Academic Press New York, Pp 89-123 (1974)

[4] PierRe R.,Fortin A. On the convergence of mixed method of Crochet and Marchal for viscoelastic flows COMP. МETH. APPL. МECH ENG. 73, PP 341-350 (1989)

[5] Y.SAAD,Brown P.N. Hybrid Krylov Methods for non linear systems of equations S.I.A.M. J Sci. StaT. Comp. 27 (1990)

[6] Touzani R. Implementation of discontinuous finite element method for hyperbolic equations Comp. Meth. IN APPLIED Mechanics AND EngeneERing 68, PP 115-123 (1998)

[7] Rallison J.M. AND HYNCH E.J. Do we understand the physics in the constitutive equation? J.N.N.F.M. 19, PP 37-55 (1988)

[8] Fortin M.,ForTIN A. A new approach for the FEM simulation of viscoelastic flows. J.N.N.F.M. 32, PP 295-310 (1989)

[9] ForTin M.,ZINE A. An improved GMRES method for solving viscoelastic flow problems J.N.N.F.M. 12, PP 1-18 (1992)

[10] Baranger J., SANDRI D. Finite element approximation of viscoelastic fluid flow; Existence of approximate solutions and error bounds NUMERISCHE MATHEMATIK 63,PP 13-27 (1992)

Remerciements : Ce travail a été réalisé dans le cadre du Groupement de Recherche "Rhéologie des polymères fondus" du CNRS.

Laboratoire D'Analyse Numérique-CNRS UMR 5585. Université Lyon 1, 43 BD. Du 11 Novembre 1918. 69622 Villeurbanne Cedex. France 